\title{
PROBLEMAS METODOLÓGICOS EN LA INVESTIGACIÓN SOBRE LAS REPRESENTACIONES MENTALES REFERIDAS A TEMAS BIOLÓGICO- NATURALISTAS EN LOS NIÑOS DE LA ESCUELA PRIMARIA
}

\author{
CARAVITAS, S. y TONUCCI, F. \\ Instituto de Psicología del CNR. Roma.
}

Comunicación presentada al Congreso Internacional «Le Fonctionnement de l'enfant à l'école». Universidad de Poitiers, 17-20 junio 1987.

(Versión đe Gené Duch, A.)

\section{SUMMARY}

The aim of this paper is to deal with the methodological problems met by the researcher when studying aspects of the child's cognitive development in order to obtain helpful information to modify the school practice.

\section{INTRODUCCIÓN}

El objetivo de este trabajo es hablar de los problemas metodológicos que el investigador encuentra cuando quiere estudiar aspectos del desarrollo cognitivo del niño, con el fin de obtener indicaciones útiles para modificar su práctica escolar ${ }^{(1)}$.

Los problemas metodológicos responden a la necesidad de coherencia entre dos aspectos relacionados en nucstro trabajo: el aspecto pedagógico y el psicológico. Desde el punto de vista pedagógico hacemos nuestro el modelo educativo que rechaza la simple transmisión de conocimientos del maestro (que es el que sabe) al alumno (que es el que no sabe), para valorizar la participación activa del nif́o en la construcción y el desarrollo de sus conocimientos. En nuestra opinión, una escuela que quiere actuar positivamente sobre el desarrollo cognitivo del niño debe partir siempre de sus conocimientos actuales de manera que los nuevos conocimientos propuestos puedan ser reconocidos por el niño como un desarrolio de sus conocimientos precedentes.

Conocer las representaciones mentales de los niños, conocer lo que ellos saben y cómo lo saben permite al enseñante comprenderlos mejor, más profundamente, y también escoger el punto de partida de sus proyectos didácticos.

Desde el punto de vista psicológico, y de acuerdo con las diferentes escuelas (Sutton, 1980; Gilbert y Watts,
1983; Driver Guesne y Tiberghien, 1985; Carey, 1985; Vicentini Missoni, 1983 (Giordan 1987), consideramos las representaciones como estructuras dinámicas, complejas y multidimensionales:

- producidas por la integración de elementos diversos: cognitivos, perceptivos, emotivos.

- construidos a través de diferentes contextos de la vida: la experiencia personal, social, escolar.

- elaborados en la memoria a través de operaciones mentales de diversos niveles de abstracción y de consciencia (el saber proposicional, el saber procesual) (Rumelhart y Norman, 1975; Anderson, 1977).

La constatación de Norman nos parece representativa: «Como expertos, nosotros tenemos el deber de desarrollar métodos experimentales apropiados, abandonando la idea de encontrar modelos mentales precisos y elegantes, aprendiendo por el contrario a comprender las estructuras messy, sloppy, incomplete, indistinct $^{(2)}$ que tiene la gente.

\section{NUESTROS OBJETIVOS DE INVESTIGACIÓN}

Como ya hemos dicho, el propósito de nuestra investigación no es la descripción completa de una tipología de conocimientos espontáneos del niño, sino la identificación de los procesos cognitivos aplicados por él 
mismo para interpretar el mundo biológico, de las relaciones entre sus representaciones mentales y la información recibida a través de los medios de comunicación social, de la relación entre dichas representaciones y los proyectos escolares y de la manera en que los procesos cognitivos se diferencian de aquellos que el docente supone.

De acuerdo con este objetivo, nosotros intentamos desarrollar una investigación que nos permita comprender:

- Las caracteristicas de las representaciones mentales en diferentes edades de niños y niñas de condiciones sociales diversas, para verificar el desarrollo y las relaciones de diversas experiencias en niños de ambos sexos que habitan en medio rural o urbano.

- La utilización por el individuo de sus propias representaciones mentales cuando recibe informaciones, cuando realiza diferentes tareas, cuando trabaja en situaciones sociales diferentes. Es decir se trata de ver «en acción» las representaciones mentales.

-Comprensión de los principios o reglas sobre los que se fundamenta la organización cognitiva de un individuo en relación con un contenido particular. Se puede probablemente remontar a la lógica natural que encuentra su aplicacion en estas inferencias y relaciones.

-Revelación de un saber tácito implícito, es decir un saber que dirige la ejecución de una tarea explícita a través de la comunicación y de la que el individuo no es totalmente consciente. No hay equivalencia entre la estructura cognitiva y su expresión exteriorizada, como tampoco hay independencia entre esta estructura y la presentación que la pone en acción.

\section{CARACTERÍSTICAS DE LA METODOLOGÍA}

Es difícil sondear la complejidad del objeto de nuestra investigación, Ios instrumentos serán siempre insuficientes para revelarla completamente.

En nuestra opinión es correcto utilizar un sistema de jnvestigación en los diferentes dominios y en los diferentes niveles de nuestro objeto de estudio. Por ello hemos preparado la experimentación teniendo en cuenta las relaciones que existen especialmente entre la ejecución de la tarea y la consigna.

\section{Relación contexto-empleo de representaciones pro- pias de los niños}

Varios autores han observado (Viennot, 1979; PerretClermont, 1980) que el contexto situacional (o la percepción que uno tiene de tal contexto) lleva a la comunicación de unas representaciones más que de otras. Si actúa en una situación del tipo sinterrogatorio escolar» o «examen» es probable que emerjan las construcciones cognitivas producidas por la ensenanza escolar. De aquí la importancia de:
- Recoger los datos fuera del contexto escolar.

- Preparar un ambiente de trabajo atrayente y estimulante para los niños.

- Proponer una organización del trabajo y de las situaciones que activen no solamente el conocimiento, sino también la habilidad y la emotividad relacionadas con el mundo animal (Tonucci, 1986).

\section{Relación tiempo-utilización de representaciones mentales}

No dedicar a la recogida de datos más que el tiempo estrictamente necesario al desarrollo de la experiencia, conduce casi siempre a pedir al nino cosas incomprensibles para él y en consecuencia, a recoger unas respues. tas difíciles de interpretar. De aquí se desprende:

- La necesidad de estar con los niños y trabajar largo tiempo con ellos, previendo momentos de juego y momentos dedicados a un mejor conocimiento de las actividades propuestas. Estar mucho tiempo con ellos no solamente cuando es necesario recoget datos, sino también y principalmente para permitir a los niños entrar en la situación experimental.

\section{Relación estímulo-movilización de las representacio- nes propias de los niños}

El término estructura cognitiva se refiere a la organización de los conocimientos en la memoria, El conocimiento incluye estructuras proposicionales, procesuales, estructuras productivas de imágenes (Anderson, 1977); estos diferentes elementos aunque integrados y relacionados entre sí en las construcciones mentales, obtienen una relevancia particular según el estímulo que los pone en acción, que dirige su recuperación en la me. moria. De aquí el interés de proponer estímulos diferenciados en diversas interacciones, partiendo de la observación directa del animal real, de su representación gráfica, de preguntas escritas, o incluso de la demanda de procedimientos.

\section{Relación modalidad de la pregunta - nivel de expli- cación de las representaciones}

La solicitud de producir ideas o razonamientos puede ser hecha a un solo individuo (por ejemplo pidiendo que se ilene una ficha, proponiendo un diálogo con un interlocutor, resolviendo un problema). En este caso el individuo está sometido a un estímulo y a la representación que él tiene del mismo.

Esta solicitud de producir ideas o razonamientos puede también ser hecha a un grupo, en esta situación las percepciones del estímulo y las respuestas al mismo pueden ser diversificadas. Estas diferentes ideas comunicadas por los elementos del grupo actúan como nuevos estímulos. Esto nos permite explorar también los "contornos potenciales» de las representaciones. Nosotros hemos considerado el grupo como un contexto «más natural» en el que se puede hacer emerger el em. 
pleo de conocimientos, así como favorecer la interacción, la comparación la metacognición (PerretClermont, 1980; Doise, 1980; Murray, 1983; Cooper. 1986).

Si nuestro objetivo es el de poner a los sujetos en las condiciones más adecuadas y naturales para resolver su tarea, el grupo y en particular la clase son considerados como el mediador social más importante, una garantía de conocimiento y solidaridad frente a la novedad de los lugares, de las personas y de las tareas. Más que pedir a los grupos que elaboren productos, se les pide que muestren, justifiquen, discutan los productos individuales en el mismo grupo, estimulando los comentarios y facilitando materia de reflexión para todos.

$\mathrm{El}$ adulto juega en el grupo un papel diferente al que jugaría si fuera un miembro del grupo, pero también muy diferente al que habitualmente realiza el enseñante en clase. Reformula la tarea, garantizando a todos su total comprensión; estimula la producción sin sugerir las respuestas; valora las intervenciones de cada niffo, particularmente las de los más «débiles»; recoge anotaciones sobre las actividades del grupo; pone a prueba las soluciones dadas, poniendo en evidencia las contradicciones. De esta manera el adulto se vuelve menos autoritario, el grupo puede escapar más fácilmente de la presión del adulto, el clima que se crea es más natural; el adulto teniendo como objetivo un trabajo autónomo y real del grupo debe aceptar también sus condiciones.

\section{Relación medios de comunicación - características de representación mentales}

La comunicación de estructuras cognitivas, su expresión pública, puede realizarse a través de la verbalización (oral o escrita en sus diferentes formas: la discusión, la compilación de fichas, descripción de fenómenos...); a través de los medios gráficos (dibujos...); a través del empleo de lenguajes formalizados... Cada uno de estos medios conecta y activa las construcciones mentales y posee reglas para «filtrar» de diferentes maneras dichas construcciones.

Se pueden establecer así relaciones entre la capacidad de empleo de reglas, eslabones de complejidad, de organización, y capacidad de reflexionar sobre las propias representaciones. De esta manera, pedir a los niños el empleo de diferentes medios de expresión nos permite evaluar las diferentes producciones obtenidas por medios diversos, su coherencia interna así como Ia gestión de esta coherencia. De forma más general nos facilita reflexionar sobre la forma en que los lenguajes «modelan» las construcciones mentales y sobre la influencia de los estereotipos culturales.

De acuerdo con estos puntos nosotros hemos proyectado un programa experimental que tiene como objetivo el estudio de cómo los niños entre 7 y 12 años co- nocen y utilizan la relación «estructura-función» en el mundo animal. Para ello hemos tenido en nuestro la boratorio una clase escolar y hemos trabajado con ella durante 4 mañanas consecutivas. El ambiente de tra bajo estaba preparado de manera que no recordaba el medio escolar, ya sea a través de la organización del espacio, de las características de las preguntas planteadas, de la forma en que los niños se sentaban, jugaban... Se pidió a los niños que trabajan individualmente, en pequeños grupos o colectivamente a nivel de toda la clase. Se han propuesto estimulos diferentes: la observación y manipulación de animales vivos, el empleo de materiales de simulación, de fichas, de problemas con varias posibilidades de respuesta, de tareas para resolver con ayuda de la fantasía... Se ha propuesto también la utilización de lenguaje verbal y escrito, la manipulación de instrumentos y la construcción con materiales reales.

Asi pues, delante de la complejidad del objeto de estudio considerado como inaccesible, nosotros hemos adoptado los «sondeos» que suscitan en los niños la producción de ideas que provienen de ópticas, de niveles, de medios diferentes de sus representaciones mentales. Un trabajo de eleboración y de interpretación correcto deberia conectar estos datos a una hipótesis interpretativa de la estructura compleja que se pretende estudiar.

La imagen de las representaciones mentales que emerge será ciertamente parcial, pero probablemente mucho más cercana a la realidad que aquella que pudiera emerger de investigaciones que, utilizando instrumentos mucho más precisos y seleccionados, consiguen circunscribir datos cuantitativos evaluables en porcentajes significativos.

\section{LAS gaRANTÍAS, LOS PROBLEMAS No RESUELTOS}

Naturalmente nadie piensa que se pueda tealizar una investigación científica correcta sin garantías suficientes de objetividad cuando disminuye:

- El valor concedido a una amplia y rápida recogida de datos limitados y precisos.

- La confianza en la elaboración de datos principalmente cuantitativos y estadísticos.

- EI valor de grupos de control en contextos como el escolar, donde cada grupo presenta elementos de variabilidad difíciles de controlar.

Surgen $\rightarrow$ cuando esto sucede- importantes problemas metodológicos que serán discutidos aquí:

\section{A. Recogida de datos durante la experiencia}

Si el interés de la investigación se desplaza de la elaboración final producida por los ninos a los procesos, se vuelve entonces necesario recoger una información re- 
lativa a las fases de esta elaboración. Cuantas más actividades se proponen a los nif̃os mayor es:

- la «dimensión» de los protocolos (notas, registros de interacción verbal, registro por vídeo de los procesos...)

- la necesidad de que otros investigadores, ausentes durante la experimentación, puedan releer e interpretar los protocolos.

- la fantasía necesaria para inventar maneras diferentes de recoger datos evitando la superabundancia de protocolos y garantizando al mismo tiempo su importancia y su significación.

- cl riesgo de interpretaciones personales.

\section{B. Personal de investigación}

Los problemas planteados piden para ser abordados la participactón en la investigación de un gran número de buenos profesionales. Cuanto más pequeñas son las garantías externas derivadas del método, más grandes deben ser las garantias que debe ofrecer el investigador. Largos entrenamientos son necesarios para limitar en lo más posible el efecto de las actitudes y de evaluaciones personales; la reflexión y la confrontación en los grupos de investigación obligan a hacer más explícitas las referencias teóricas, así como el sistema de valores y de convicciones con los que se realiza la interpretación de datos. Las representaciones mentales del investigador son filtros tan selectivos como las metodologías utilizadas. Una composición multidisciplinar del grupo de investigación, como en nuestro caso, comporta necesariamente dedicar más tiempo a la confrontación, la negociación y la integración de puntos de vista, tendiendo a una homogeneidad de criterios y de actitudes. En cada una de nuestras sesiones experimentales eran necesarios, por ejemplo cinco operadores uno por cada pequeño grupo de la clase y para la coordinacion general - y estos cinco se transformaban en nueve cuando era necesario tomar nota en los grupos de trabajo.

\section{Elaboración}

Un aspecto particularmente difícil de la interpretación de informaciones es la comparación horizontal de los datos producidos por un mismo sujeto, comparación que es necesaria para evaluar la amplitud, la articulación, y la densidad de las representaciones estudiadas. No es fácil comparar productos tan diferentes (diálogos, fichas, dibujos, construcciones) encontrándose categorías, criterios comunes, que permiten comprender las estructuras mentales que las han producido. Es más fácil sacar a la luz la diversidad o la consistencia de estas estructuras comparando los resultados obtenidos en tareas puntuales con toda una población experimental o por grupos de edades diferentes. Probablemente los resultados asi obtenidos permitirán examinar con una seguridad suficiente los datos individuales.

\section{Implicación en la experiencia}

Largos periodos de trabajo con los niños, el deseo de ponerles en condiciones más favorables, la significación de las proposiciones y el deseo de seguir y comprender sus procesos, hace diffcil al investigador el mantenimiento de un papel standard, distanciado y objetivo.

La implicación es fatal y es preciso tenerla en cuenta en la evaluación de la experimentación.

\section{F. Comunicación de resultados}

A las personas implicadas en este tipo de investigaciones, no les es siempre fácil encontrar canales de intercambio, sea con los colegas en el debate científico in. ternacional, o con el mundo escolar que es el destina. tario natural de estas investigaciones. Es dificil relatar al mundo científico los resultados de estas investigaciones con la brevedad que solicitan (y que tal vez es necesaria) las revistas internacionales, no pudiendo con * tar más que marginalmente con la ayuda de representaciones cuantitativas, esquemáticas de datos. Es difícil por otra parte comunicar con los enseftantes, ya sea porque el sentido del trabajo de los investigadores está relacionado con un marco cultural que actualmente no forma parte de la formación inicial de nuestros enseñantes, ya sea porque los resultados de las investigaciones se traducen difícilmente en actividades y comportamientos didácticos. Su objetivo es por el contrario una modificación de actitudes implicitas del enseñante (de cómo hacer más de lo que hace), pero es difícil persuadir al enseñante para que reconozca esta necesidad.

\section{Notas}

(I) Conectando asi con el debate abierto en el Seminario de Roma en 1980 y recogido en: Tonucci, F., Caravita, S., Detti. E. (eds) (1981) La ricerca nella scuola di base Roma. Instituto de Psicologia.

(2) Podemos traducir estos términos por: confusas, inconsistentes, incompletas y poco precisas. 


\section{REFERENCUAS BIBLIOGRÁFICAS}

ANDERSON, R.C., 1977, The notion of schemata and the educational entreprise general discussion of the conference. In: Anderson R.C., Spiro R.G., Montague W.E. (eds), Schooling ald acquisition of knowlege, LEA, Irillsdaie.

CHAMPAGNE, A.B., KLOPFER, L.E., DESENA, A.T. and SQUIRES, D.A., 1981, Structural representation of students' knowicdge before and after science instruction. $J$. Research in Science Teaching, 18 (2), 97-111.

COOPER, R., MARQUIS, A. and EDWARD, D., 1986, Four perspectives on peer learning among elementary school chiidren. In: Mueller E.C., Cooper C.R., Process and outcome in peer relationships, Acad. Press. Orlando.

DOISE, W., MACKIE, D., 1981, On the nature of social cognition. In: Forges J.P. (ed), Social cognition perspective on everyday understanding, Acad. Press., p. 53. London.

DRIVER, R., GUESNE, E. and TIBERGHIEN, A., 1985, Children's ideas in science, Milton Keynes. Philadelphia.

GILBERT, J.K., WATTS, D.M., 1983, Concepts, misconceptions and alternative conceptions changing perspectives in science education, Studies in Science Education, 10, p. 61-98.

GIORDAN, A. (ed), 1987, L'élève et/ou les connaissances, scientifiques Peter I ange. Berne.
MURRAY, F.B., 1983, Learning and development through social interaction and conflict a challenge to social learning theory. In: Liben L. (ed), Piaget and the foundation of knowledge, Erlbaum, Hillsdale.

NORMAN, D.A., 1983, Some observations on mental models. In: Gentner D., Stevens A.L., Mental models. LEA, p. 7.14. Hillsdale,

PERRET-CLERMONT, A.N., (ed), 1980, Social interaction and cognitive development in children, European Monographs in Social Psychology. Acad. Press. London.

RUMELHAR $\Upsilon$, D.E., NORMAN, D.A., 1975, The active structural network, In: Norman D.A., Rumelhart D.E., Explorations in cognition, Fraeman. San Francisco.

SUTTON, C.R., WEST, L., (eds), 1982, Investigating children's existing ideas about science, Univ. Leicester, School of Education. Leiccster.

TONUCCI, F., 1986, A laboratory for the study of cognitive process in science teaching during compulsory education, European Journal of Psychology of Education, 1 (1), pp. 101-106.

VICENTINI-MISSONI, M., 1983, Conoscenza scientifica c conoscenza comune, in Conoscenza scientifica e insegna. mento, Loescher, pp. 21-63. Torino.

VIENNOT, L., 1979, La raisonnement spontané en dynamique élémentaire, Paris, Hermann. 